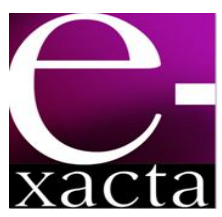

ISSN: 1984-3151

\section{RECOMENDAÇÃO DE ITENS DE INFORMAÇÃo SENSÍVEL AO ESPAÇO}

\author{
RECOMMENDATION OF INFORMATION'S ITEMS SPACE’S SENSITIVE
}

\section{Ariosvaldo Ribeiro Merlo; Italo Paolo Saturnino de Jesus²; Joaquim Jose da Cunha Junior ${ }^{3}$}

1 Bacharel em Ciência da Computação. Centro Universitário de Belo Horizonte - UniBH, 2012. Belo Horizonte, MG. arioswaldomerlo@gmail.com.

2 Bacharel em Ciência da Computação. Centro Universitário de Belo Horizonte - UniBH, 2012. Belo Horizonte, MG. italopsj@gmail.com.

3 Mestre em Engenharia de Produção. PPGEP/UFMG, 2012. Professor do Centro Universitário de Belo Horizonte UniBH. Belo Horizonte, MG. joaquim.jose@prof.unibh.br.

Recebido em: 09/03/2013 - Aprovado em: 20/04/2013 - Disponibilizado em: 11/06/2013

RESUMO: Este artigo apresenta um estudo de aplicação da recomendação colaborativa de dados sensível ao espaço, baseado na experiência de uso de um aplicativo mobile, na plataforma Android, para recomendação dos dados, podendo ser utilizados os comentários e qualificação de itens, gerados por usuários do aplicativo desenvolvido. É possível, dessa forma, usar filtros como "de proximidade", "mais comentado", "melhor avaliado" e "ordem alfabética".

PalavRa-Chave: Android. Recomendação. Banco de dados. Sensível ao espaço. Filtragem colaborativa.

ABSTRACT: This article presents a study of applying collaborative recommendation sensitive data space, based on the experience of using a mobile application on the Android platform. For recommendation of the data can be used comments and qualifying items generated by users of the application developed. Can use filters such as proximity, most talked about, best rated and alphabetically.

KEYWORD: Android. Recommendation. Database. Space-sensitive. Collaborative filtering.

\section{INTRODUÇÃO}

O crescimento da internet trouxe vários benefícios. $\mathrm{O}$ aumento da quantidade de fontes de informação e conteúdos proporcionou ao usuário diversas opções de referências. Contudo, esse excesso trouxe um verdadeiro caos de informação, pois a ausência de fontes oficiais e confiáveis pode confundir o usuário.
Para minimizar as dúvidas e necessidades frente a tantas alternativas, o usuário, geralmente, confia nas recomendações que são passadas por outras pessoas (REATEGUI; CAZELLA, 2005).

Os sistemas de recomendação auxiliam no aumento da capacidade e eficácia do processo de indicação e recomendação. Um dos grandes desafios desse tipo de sistema é realizar o casamento correto entre o que está se recomendando e quem está recebendo a 
recomendação, ou seja, definir e descobrir este relacionamento de interesses (REATEGUI; CAZELLA, 2005).

Este trabalho apresenta um estudo sobre recomendação de itens e o desenvolvimento de um aplicativo para dispositivos móveis baseado em banco de dados e recuperação de informação e tem como objetivo geral desenvolver um aplicativo móvel que utilize os princípios de recuperação de informação e banco de dados para gerar informações de recomendação de itens baseado no perfil de cada usuário do aplicativo.

Os objetivos específicos são o desenvolvimento de um aplicativo para a plataforma Android, a recuperação de informações armazenadas em um banco de dados na web e a recomendação destes itens aos usuários do aplicativo com base em sua localização geográfica e perfil do usuário.

O desenvolvimento do artigo auxilia no embasamento teórico e serve como fonte norteadora da ideia central do aplicativo.

O que motivou o interesse em realizar tal trabalho foi a possibilidade de desenvolver um aplicativo voltado para as necessidades diárias dos usuários, tornando-o público, de livre utilização, o que poderia ser útil para a vida de várias pessoas.

O presente trabalho encontra-se dividido em seis seções. Após esta breve introdução, tem-se a segunda seção, que apresenta a metodologia utilizada para no desenvolvimento. $\mathrm{Na}$ terceira seção é abordado o tema central do estudo, onde são explicados os tipos de sistemas de recomendação e suas técnicas. A quarta seção apresenta o desenvolvimento do aplicativo, as ferramentas utilizadas, a estrutura do banco de dados, algumas funcionalidades implementadas e o versionamento mínimo do sistema Android que comporta o aplicativo. $\mathrm{Na}$ quinta seção é feita uma análise dos resultados obtidos e da alcançabilidade, além de serem sugeridas novas funcionalidades que podem enriquecer ainda mais a experiência de usabilidade do aplicativo. Por fim, a última seção apresenta a conclusão do trabalho.

\section{Metodologia}

Foram utilizadas como metodologia de pesquisa abordagens qualitativas, para aplicar um algoritmo eficiente de recuperação de informação e de recomendação de itens para a aplicação desenvolvida, pesquisas exploratórias por meio dos levantamentos bibliográfico, documental e visual vinculados ao tema e à plataforma escolhida. Para auxiliar no desenvolvimento desta aplicação mobile foram utilizados materiais de autores que possam ser confiáveis e fóruns para buscar soluções e dicas para alguns problemas encontrados durante 0 desenvolvimento. Por meio de todas as informações e conhecimentos adquiridos foi possível 0 desenvolvimento do aplicativo e deste artigo.

\section{SISTEMAS DE RECOMENDAÇÃO}

Uma recomendação pode se basear nas preferências de outro usuário, que ao efetuar uma avaliação, seja positiva ou negativa, o sistema pode recomendar este item, e assim direcionar a um indivíduo específico, como, por exemplo, sugestões a um amigo ou a um público mais geral, com uma crítica de um especialista veiculada em algum meio de comunicação. Para quem recebe a recomendação, ela funciona como um filtro que auxilia em sua escolha entre as opções disponíveis (SAMPAIO, 2007).

Segundo o mesmo autor, são chamados de sistemas de recomendação os sistemas de informação que automatizam ou dão suporte computacional a algum processo de recomendação, como coleta, armazenamento e retorno de informações, como 
ilustra a Figura 1. Eles podem fornecer recomendações aos usuários com base em seus interesses e levando em consideração as opiniões de outros usuários.

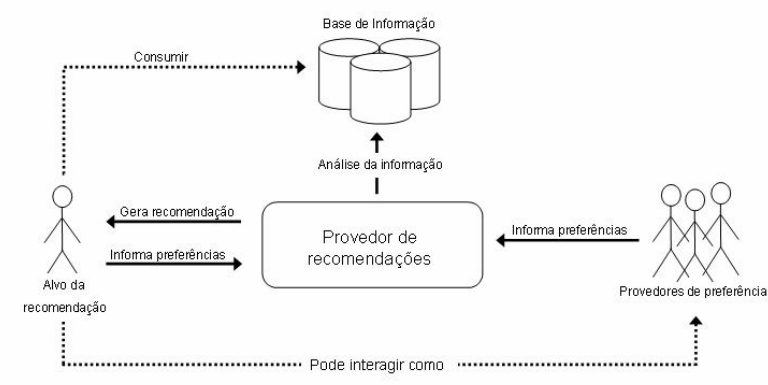

Figura 1 - Modelo do processo de recomendação Fonte - SAMPAIO, 2007, p. 10.

\subsection{Sistemas DE RECOMENDAÇÃo NÃO PERSONALIZADA}

O papel principal destes sistemas é o de mediador entre os geradores e consumidores de recomendação, fazendo apenas a coleta e a distribuição das recomendações (SAMPAIO, 2007).

Nos Sistemas de Recomendação Não Personalizada, os usuários avaliam o conteúdo através de comentários positivos, negativos ou neutros, e outros usuários se baseiam nesses comentários e podem fazer sua própria avaliação sobre o conteúdo (SAMPAIO, 2007).

A Figura 2 exemplifica os comentários em um sistema de recomendação não personalizado, no qual são exibidos com o marcador verde os comentários positivos, com azul os neutros e vermelho os comentários negativos sobre o conteúdo.
Qualificações recebidas pela venda deste produto

Ver qualificaçōes Todos

$\oplus 10$ de Maio (PANICO

"O produto chegou ok. Recomendo o vendedor !"

- 10 de Maio (MARCELOELIAS47 ...)

"nenhum comentário"

๑ 10 de Maio (DAIANEPRAN..)

"Ótima negociação, retirei todas as minhas duvidas com exito.Aparelho chegou

em ótimo estado, lacrado, e antes mesmo do que imaginava.Parabéns !!!"

$\vartheta 10$ de Maio (Joseniltonlima JU,

"comprei dois aparelhos, um veio funcionando perfeito e o outro não está

funcionando, assim que eu mandar apara garantia tudo certo ai mudo a

qqualificação."

Ver todas as qualificações

Figura 2 - Qualificações de conteúdo

Fonte - mercadolivre.com.br

\subsection{SISTEMAS DE RECOMENDAÇÃO PERSONALIZADA}

Os Sistemas de Recomendação Personalizada são capazes de aprender e identificar as necessidades individuais de um usuário e armazenar essas preferências, gerando assim um perfil de usuário, de forma que se possa fazer recomendações mais adequadas às suas necessidades e gostos (SAMPAIO, 2007).

Segundo o mesmo autor, esses sistemas utilizam técnicas de filtragem de informação e aprendizagem de máquina para gerar as recomendações apropriadas para cada perfil de usuário. As técnicas de filtragem podem ser divididas em Filtragem Baseada em Conteúdo, Filtragem Social ou Colaborativa e Filtragem Híbrida, que serão melhor abordadas nas próximas seções.

\subsubsection{Filtragem BASEADA EM CONTEÚDO}

Esse tipo de filtragem recomenda itens ao usuário, baseando-se na semelhança com outros que ele já tenha demonstrado interesse em algum momento. 
Uma das formas de utilizar esse tipo de filtragem é através de avaliações dos itens feitas pelo usuário. Uma vez que a avaliação é feita, o sistema busca itens que tenham determinado grau de semelhança, quanto ao conteúdo, com aqueles já classificados pelo usuário como de seu interesse. Os itens que não se assemelham com o perfil traçado são descartados para esse usuário (REATEGUI; CAZELLA, 2005).

Os sistemas de filtragem baseados em conteúdo apresentam algumas limitações, por exemplo: conteúdos de dados pouco estruturados geram maior dificuldade de serem analisados. A utilização de sinônimos no texto descritivo dos itens é outro problema encontrado na hora de classificar o item como semelhante ao de interesse do usuário ou não (REATEGUI; CAZELLA, 2005).

\subsubsection{Filtragem Colaborativa ou Social}

A técnica de Filtragem Colaborativa baseia-se nas avaliações de outros usuários que tenham perfis similares para fazer a melhor recomendação para o usuário.

Segundo Sampaio (2007), os sistemas de filtragem colaborativa identificam usuários similares e recomendam os itens melhor avaliados por eles a um usuário-alvo. O processo de recomendação está totalmente ligado à similaridade dos usuários, ao contrário dos sistemas baseados em conteúdo, que se baseiam na similaridade dos itens.

Ainda segundo o mesmo autor, o processo de filtragem colaborativa é dividido em três passos:

- Representação dos dados de entrada: o usuário expressa suas preferências avaliando itens do sistema. Essas avaliações, positivas ou negativas, mostram o interesse do usuário em itens específicos e são armazenadas em seu perfil;
- Formação de vizinhança: o sistema compara o perfil do usuário-alvo com os de outros usuários do sistema, de forma que consiga encontrar perfis similares para formar a "vizinhança" do usuárioalvo;

- Geração de recomendação: baseado nas avaliações feitas pelos componentes da vizinhança, o sistema gera a recomendação para o usuário-alvo, ou seja, o sistema recomenda-Ihe os itens que seus vizinhos mais gostaram.

Nos sistemas de Filtragem Colaborativa não há a necessidade de representar informações sobre o conteúdo dos itens, basta apenas um identificador para cada item e outro para cada usuário (SAMPAIO, 2007).

\subsubsection{FILTRAGEM HíBRIDA}

A Filtragem Híbrida surgiu para tirar proveito das vantagens existentes na Filtragem Baseada em Conteúdo e na Filtragem Colaborativa, deixando de lado as desvantagens encontradas em ambas (SAMPAIO, 2007).

\section{Desenvolvimento do Aplicativo}

Para a escolha de qual sistema móvel - no qual o aplicativo seria desenvolvido - foram utilizadas informações de mercado, para verificar qual deles tinha maior abrangência. Segundo Camargo (2011), em relatório da Canalys, uma respeitada empresa de pesquisa de mercado, o Android OS foi responsável por $48 \%$ do mercado de smartphones no segundo trimestre de 2011 e apresentou a maior taxa de crescimento em relação à concorrência. Outro dado importante é a grande quantidade de fabricantes de smartphones que utilizam o sistema Android, abrangendo vários modelos de aparelhos em diferentes faixas de preços. 
Ao se levantar os requisitos para o desenvolvimento do aplicativo, houve a necessidade da criação de um CRUD (criação, leitura, atualização e deleção, em língua portuguesa), para aprimorar o armazenamento e recuperação das informações. Fez-se necessária também a utilização de um Banco de Dados por meio da ferramenta MySQL, para armazenar dados dos usuários e informações sobre os itens que estarão no programa de recomendação.

Para a integração do banco de dados com o aplicativo está sendo utilizado um Web Service, que é uma ferramenta que faz a interligação entre plataformas que não se comunicam diretamente. O Algoritmo 1 é um exemplo do trecho de código utilizado para a criação de um usuário no banco de dados utilizando Web Service em PHP.

Algoritmo 1 - Algoritmo de criação de usuário do aplicativo no banco de dados

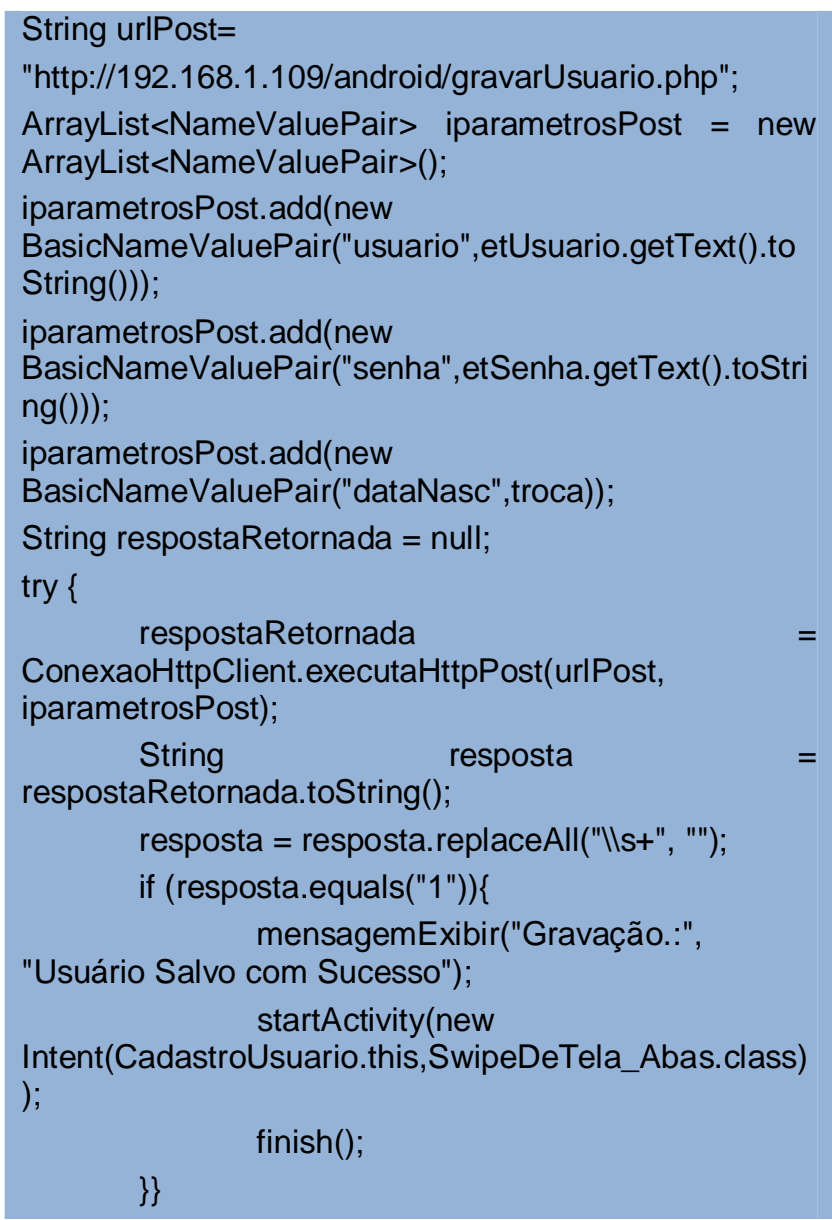

\subsection{Conjuntos de Ferramentas}

Para o desenvolvimento do aplicativo na plataforma Android, foi utilizada a IDE (Ambiente Integrado de Desenvolvimento, em língua portuguesa) MOTODEV Studio for Android 4.1, que auxilia na geração do código e integração com a interface do sistema Android. A base de dados desenvolvida está inclusa no conjunto de ferramentas do XAMPP, que possui o suporte de um banco de dados MySQL e a linguagem de programação PHP, utilizada para gerar 0 WebService, o qual é um meio de integração entre o Android e a base de dados.

\subsection{TESTES COMPUTACIONAIS PRELIMINARES}

Com o desenvolvimento do CRUD, da interface em Android, do banco de dados no MySQL (Figura 3) e do WebService em PHP, foram possíveis os testes de interface.

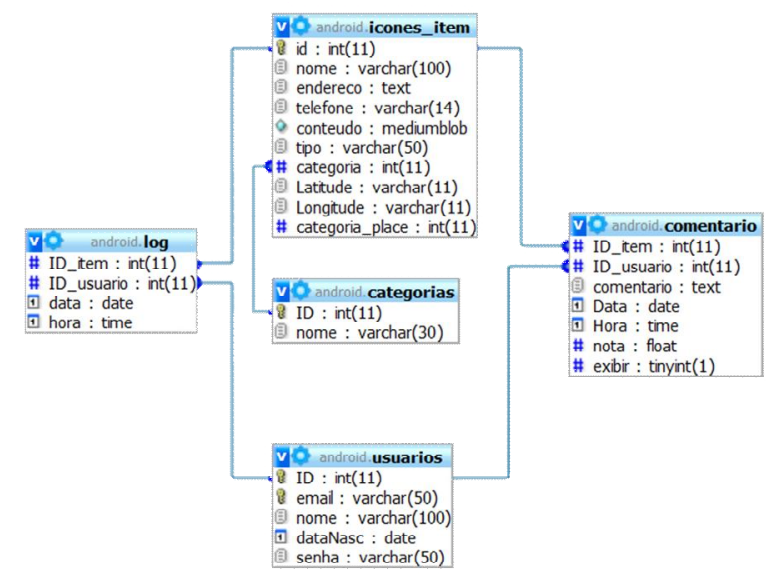

Figura 3 - Modelo ER do banco de dados

Com a validação dos dados como data de nascimento anterior à data atual, senha idêntica ao do campo de confirmação de senha e e-mail, foi possível inserir os dados do usuário, na respectiva base, e recuperar esses mesmos dados para a validação de login, por meio do WebService. Esses testes foram realizados por meio de um emulador e outros dois dispositivos móveis com sistema Android, de marcas e modelos 
distintos, utilizando como versão básica de desenvolvimento do aplicativo o sistema Android 2.2, conhecido também como FROYO.

\subsection{TESTES COMPUTACIONAIS INTERMEDIÁRIOS}

Após os testes preliminares com o CRUD, WebService e com o Banco de Dados, foram realizados os testes intermediários para recuperação dos dados e imagens dos itens, sendo estes exibidos na tela do dispositivo Android, como mostra a Figura 4.

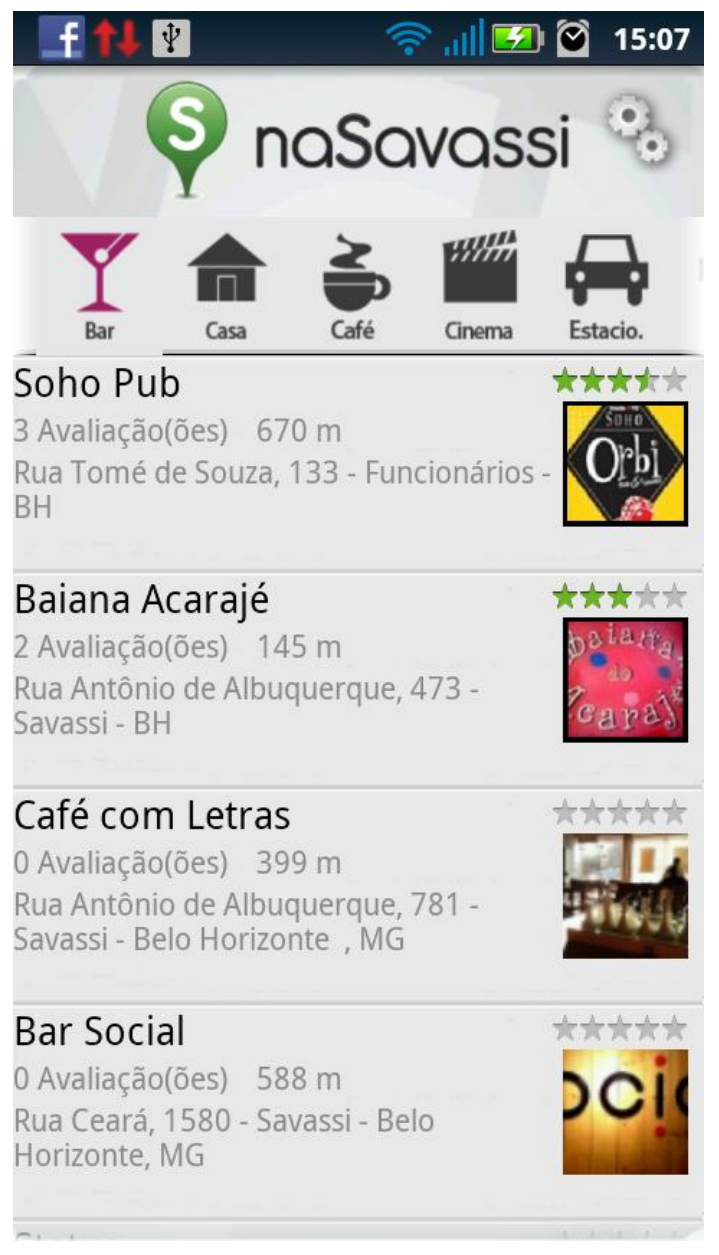

Figura 4 - Itens de exibição

A exibição desses dados é tratada pelo código XML do Android e a inserção dos identificadores únicos de cada item, para persistência dos dados, pelo código JAVA do Android, para permitir a criação de comentários e interligação das telas com a recuperação desses dados, sem a necessidade de outro acesso ao WebService. Tais testes foram realizados por meio de um emulador com versão do Android 2.2 e 4.0, e os dois dispositivos móveis com sistema Android 2.3, de marcas e modelos distintos, mantendo ainda como versão básica de desenvolvimento do aplicativo o sistema Android 2.2, para manter compatibilidade com a maioria dos dispositivos móveis.

\subsection{TESTES COMPUTACIONAIS FINAIS}

Com todo o desenvolvimento das partes estruturais da recuperação de informações na web pronto, foi criado um banco de dados local (no Android). Nele são armazenadas as informações a respeito dos itens a serem exibidos para o usuário, para evitar que o aplicativo fique acessando a internet a cada mudança de tela, reduzindo, assim, o consumo de dados pela rede $3 G$ do celular, o que gerou uma melhora, em termos de desempenho, na exibição das informações.

Pensando em auxiliar o usuário quanto a sua posição geográfica em relação aos locais, é exibida na tela a distância do usuário em relação a cada local, como mostrado na Figura 4. Para isso, são armazenadas, juntamente com as informações do local, suas coordenadas, e o próprio aplicativo, utilizando as coordenadas atuais do celular, calcula uma distância aproximada até o referido local.

Para a recomendação foi gerado um menu de configurações onde o usuário pode escolher a forma como os dados serão exibidos na tela, o que pode ser visto na Figura 5. 


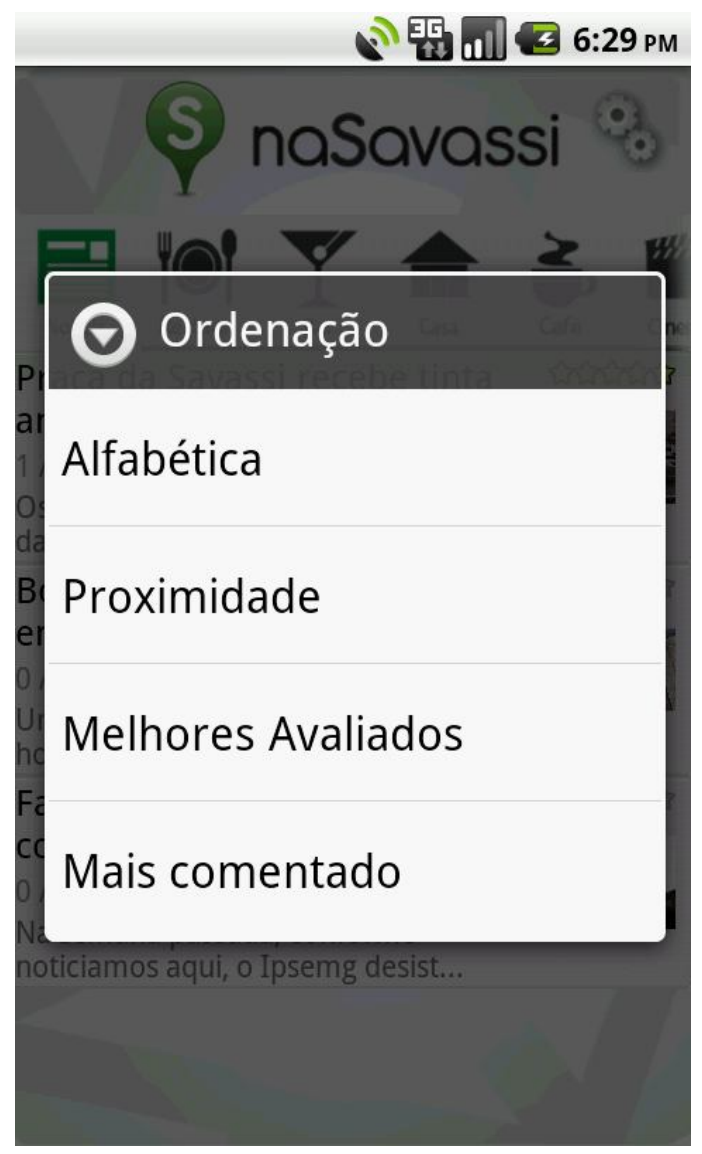

Figura 5 - Menu de ordenação

\section{ANÁLISE dOS RESULtAdOS}

Com o aplicativo em fase final de desenvolvimento pôde-se constatar que alguns dos objetivos foram alcançados. A implementação da recomendação baseada em geolocalização foi alcançada e, a partir dos testes realizados, concluímos que os resultados são satisfatórios, visto que o cálculo da distância é feito com base na distância em linha reta entre dois pontos, e para traçar o melhor caminho foi utilizado o aplicativo Mapas da Google, nativo no sistema Android.

Outro resultado obtido até 0 momento foi a recomendação de itens baseada na avaliação de outros usuários utilizando a técnica de Filtragem Colaborativa. Segundo esta, os itens com melhor avaliação são listados preferencialmente em relação a itens com nota média mais baixa.
Por outro lado, ainda não está sendo feita a recomendação baseada nos perfis de usuários, pois a base de dados não contém informações de usuários e avaliações suficientes para que se possa definir perfis de usuários. Para que este objetivo possa ser alcançado, será lançada uma versão do aplicativo, intitulada "Versão Beta" - que será a primeira versão do aplicativo e que já permite que os usuários utilizem normalmente as suas funcionalidades - e servirá para coletar os dados necessários para que nas próximas versões do aplicativo seja feita a recomendação com base no perfil de cada usuário, personalizando-a.

\section{Conclusão}

A partir desse estudo foi possível verificar quais as técnicas podem ser empregadas em um mecanismo de recomendação, como é seu funcionamento, sua importância e como desenvolver um aplicativo utilizando suas técnicas.

Com o desenvolvimento do aplicativo, e visando conquistar mercado, notou-se a importância de se fazer uma recomendação mais próxima e personalizada para os grupos de usuários, pois isso pode motivá-los a utilizar o aplicativo e indicar este para outros usuários, tornando-o, assim, cada vez mais popular, que é o objetivo, pensando em termos mercadológicos.

Dentre as dificuldades encontradas no desenvolvimento deste projeto pode-se destacar a inicial falta de conhecimento na linguagem Android, o que poderia ser um impeditivo para o prosseguimento do desenvolvimento do aplicativo.

Uma perspectiva futura para o aplicativo é incluir a recomendação por perfil de usuário, visto que já é um processo desejado e em andamento. Outra funcionalidade a ser desenvolvida futuramente é a recomendação não somente dos locais, mas de 
especialidades ou promoções oferecidas pelos locais, podendo utilizar a lógica de compra coletiva (cupons).

Por fim, por se tratar de um aplicativo hiperlocal (ou seja, limitado a uma região específica), uma possibilidade futura seria ampliar sua área de abrangência, desenvolvendo versões específicas para outras localidades, inicialmente na região metropolitana de Belo Horizonte.

\section{Agradecimentos}

Os autores agradecem primeiramente a Deus, por nos dar força para sempre seguir em frente. Ao professor Wladmir Cardoso Brandão e ao naSavassi, pela oportunidade e confiança para a realização deste trabalho. A todos que contribuíram direta e indiretamente, muito obrigado.

\section{REFERÊNCIAS}

CAMARGO, Sergio. O Android em alta no mercado mundial, 2011. Disponível em: <http://www.fandroid.com.br/2011/08/04/o-android-emalta-no-mercado-mundial/>. Acesso em: 15 mai. 2012.

MERCADO LIVRE. Disponível em: <mercadolivre.com.br>. Acesso em: 09 mai. 2012.

REATEGUI, Elisio Berni; CAZELLA, Sílvio César. Sistemas de Recomendação, 2005. Disponível em: <http://scholar.googleusercontent.com/scholar?q=cach e:FXRXKpeLaC8J:scholar.google.com/+recomenda\% C3\%A7\%C3\%A3o+de+itens+na+internet\&hl=ptBR\&as_sdt=0,5>. Acesso em: 23 mar 2012.

SAMPAIO, Igor Azevedo. Aprendizagem Ativa em Sistemas de Filtragem Colaborativa, 2007. Disponível em:<http://www.cin.ufpe.br/ rbcp/dissertacoes/disserta caolAS.pdf >. Acesso em: 09 mai. 2012. 